\section{Alcohol-related deaths: Birmingham Coroner's records 1980}

The consumption of alcohol (ethanol) can contribute to a number of different causes of death, as shown by the Coroner's records in Birmingham for 1980.

\section{Subjects, methods, and results}

During 1980, 589 inquests were completed of which $119(20 \%)$ were on people whose deaths were related to the consumption of alcohol. Verdicts of alleged homicide, road traffic accidents, suicides, open verdicts, and various "others" were related to alcohol in $32 \%$ of cases (see table). Among the 305

Number of deaths recorded by Birmingham Coroner in 1980 which were related to alcohol consumption

\begin{tabular}{lccc}
\hline & Total No of & \multicolumn{2}{c}{ Alcohol-related deaths } \\
\cline { 3 - 5 } deaths & No & $\%$ \\
\hline Alleged homicide & 27 & 17 & 63 \\
Various "other" & 23 & 8 & 35 \\
Road traffic accidents & 111 & 38 & 34 \\
Open verdict & 27 & 8 & 30 \\
Suicide & 96 & 20 & 21 \\
\hline Total & 284 & 91 & 32 \\
\hline & & & \\
Home accidents & 165 & 19 & 12 \\
Accidents elsewhere & 57 & 7 & 12 \\
Natural causes & 72 & $2 *$ & 0 \\
Work accidents & 11 & 0 & 9 \\
\hline Total & 305 & 28 & 20 \\
\hline Grand total & 589 & 119 & \\
\hline
\end{tabular}

*Alcoholic liver cirrhosis

deaths which were due to accidents at home, at work, and elsewhere, as well as to natural causes, significantly fewer (Student's $t$ test, $p<0.001$ ) were related to alcohol consumption ( $9 \%)$. Verdicts of homicide included murder, manslaughter, infanticide, and causing death by reckless driving. Eight deaths (various "other") resulted from self-neglect due to alcoholism (one), drug addiction from morphine and alcohol (one), and acute alcoholic intoxication (six). Thirteen of the 38 victims of road traffic accidents were intoxicated pedestrians. Open verdicts were given where there was insufficient evidence for a more precise verdict.

To identify the type of person most likely to die from alcohol-related incidents, I excluded the 17 victims of another person's alcohol abuse (13 road accidents, four homicides) from further analysis. Of the remaining 572 deaths, 102 were alcohol-related : $90(27 \%$ of 330$)$ occurred in men and significantly fewer $(p<0.001)$ in women $(12 ; 5 \%$ of 242 deaths). More death $(p<0.001)$ were related to alcohol among young people aged 15 to 34 years $(48 / 122,39 \%)$ than among those over 35 years $(54 / 413,13 \%)$. Similarly, divorced or separated people $(24 / 54,44 \%)$ were more likely to be victims of alcohol-related accidents $(\mathrm{p}<0.001)$ than married and single people combined $(72 / 367,20 \%)$ or widows $(6 / 151,4 \% ; 0.01<p<0.02)$

Of 58 deaths in people born in Ireland or Scotland, $26(45 \%)$ were related to alcohol, which was significantly higher $(p<0.001)$ than for those born elsewhere $(15 \%)$. This group was significantly $(p<0.02)$ older (mean age 48 ) and more likely $(p<0.001)$ to be married $(45 \%)$ compared with all the other victims of alcohol-related deaths (mean age 38 ; married, $21 \%$ )

A further 69 deaths occurred as complications of chronic alcoholismincluding liver cirrhosis, cardiomyopathy, pancreatitis, hepatitis, and dementia - and required no inquest. These subjects were older (mean age 55) and more were women ( $25 \%$ ) compared with the 102 alcohol-related victims identified at inquest. Eight (12\%) were Indian-born Sikhs, who comprised an estimated $4 \%$ of the Birmingham population.

\section{Comment}

The recorded total number of $\mathbf{1 8 8}$ alcohol-related deaths is probably an understatement either because of lack of conclusive evidence or because of failure to report. Clearly, violent death is frequently related to alcohol. Young men and divorced or separated people, together with the Irish and Scots, seem particularly vulnerable to alcohol-related accidents.

The difficulties for the police are obvious, since three out of four people in their custody who died were drunk. Six out of 10 overdoses from dextropropoxyphene were associated with alcohol consumption, so confirming the danger of this combination. ${ }^{1}$ Ethnic groups vary in their susceptibility to alcohol abuse; perhaps Sikhs, as well as the Irish and Scots, are prone to alcohol-related disorders. The 11 deaths from acute alcoholism (five classified as accidents) emphasise the gravity of this medical emergency.

The role of alcohol in suicide is in reducing self-restraint and in potentiating other poisons. Prolonged dependence on alcohol may also create an intolerable state of isolation and despair. Surprisingly, in 1980 and over the preceding four years no fatal accidents at work were related to alcohol consumption.

I am grateful to $\mathrm{Mr}$ A B Neale, chief statistician, Birmingham City Council, for advice and statistical help in preparing this paper.

1 Carson DJL, Carson ED. Fatal dextropropoxyphene poisoning in Northern Ireland. Lancet $1977 ; \mathrm{i}: 894-7$.

(Accepted 18 fanuary 1982)

HM Coroner's Court, Birmingham B4 6NE

R M WHITTINGTON, BM, DMJ, coroner

\section{Ischaemia after use of finger tourniquet}

Finger tourniquets are widely used in minor surgical procedures in accident and emergency departments and by general practitioners. We report a case of ischaemia after the use of a finger tourniquet and suggest some precautions that might be taken.

\section{Case report}

A 34-year-old man presented to the accident and emergency department with a wood splinter in the soft tissues of his left index finger. He was non-smoker and had previously been healthy. Removal was undertaken when a digital nerve block had taken effect and a tourniquet been applied. The digital nerve block was achieved by injecting $1 \mathrm{ml} 2 \%$ lignocaine without adrenaline into the web spaces on each side of the finger. The tourniquet used was a strip of latex rubber, which was wound around the finger from the tip proximally; at the base of the finger the strip was secured by an artery forcep. This exsanguinated the finger in addition to occluding the blood supply.

The tourniquet remained in place for 20 minutes. When it was removed the finger remained ischaemic distal to the proximal interphalangeal joint. The patient was admitted to hospital and treated by intravenous infusion of $500 \mathrm{ml}$ low-molecular-weight dextran (Lomodex 40); he was also given dipyridamole $10 \mathrm{mg}$ intravenously followed by $150 \mathrm{mg}$ six-hourly, and alcohol in the form of whisky. While these measures were being taken his finger was packed in ice and his arm warmed. Circulation improved over 12 hours and he was discharged the next day. Review two weeks later showed complete recovery.

\section{Comment}

Tourniquets at the base of the digit are widely used in minor operations on both fingers and toes. The technique of combining exsanguination with the use of a tourniquet at the base of a finger has been described by several authors, who regard the technique as safe. ${ }^{12}$ Others, however, disagree, ${ }^{3}$ though there is little recorded evidence of their reasons. We perform at least 1000 operations a year using such tourniquets, and this is the first complication we have observed.

With a finger tourniquet, unlike the pneumatic tourniquet, there is no way of measuring the pressure applied to the tissues. The most common cause of complications after the use of tourniquets is probably excessive pressure owing to faulty gauges rather than prolonged application." It is important that the finger tourniquet should be tightened only enough to occlude the blood supply. The pressure on the neurovascular bundle will be further increased if a large quantity of local anaesthetic is injected into the base of the finger after the tourniquet has been applied. In our case injection was into the web space, but it may be safer to insert the local anaesthetic into the metacarpal canal. Local anaesthetic should not contain adrenaline: the hazards of injecting adrenaline into fingers and toes are well recognised. ${ }^{5}$

If the finger is to be completely covered by a dressing this should not be applied until the tourniquet has been removed and the circula- 